\title{
Transcranial direct current stimulation in dementia: a possible breakthrough?
}

\section{Introduction}

Dementia is associated with substantial economic and societal costs, as its worldwide cost in 2015 was estimated to be (US) $\$ 818$ billion, an increase of $35 \%$ since $2010 .{ }^{1}$ While there are a number of drugs to manage some of its behaviorial symptoms, pharmacological treatment only has a limited degree of efficacy, including undesirable side effects; ${ }^{2}$ in this sense, it is crucial to develop additional treatments. As such, there is a growing interest in non-pharmacological approaches for frontotemporal dementia (FTD), which does not have any specific pharmacological intervention. One area that has recently garnered considerable clinical and research interest in this field is the use of non-invasive brain stimulation.

Transcranial direct current stimulation (tDCS) is a non-invasive neuromodulation technique that delivers a low electrical current, through two scalp electrodes, which induces bi-directional polaritydependent changes in the cerebral cortex. It attempts to facilitate focal, prolonged shifts in cortical excitability at or around the time stimulation is provided. Typically, an anodal and cathodal electrode is placed on the scalp in accordance with the International 10-20 system. $^{3}$ This intervention is considered safe and noninvasive, as it does not involve implantation, injection, or any skin penetration. ${ }^{4}$ Different electrode configurations are believed to affect the focality of stimulation and the path of current flow. The electrical currents of tDCS are believed to modulate neuronal resting membrane potentials, which occur in a bidirectional radial manner between electrodes. Cathodal tDCS is correlated with decreased cortical excitability due to subthreshold incremental hyperpolarization of membrane potentials. Anodal tDCS (a-tDCS), in which the positively-charged electrode is placed over the targeted cortical region, is thought to lead to increased cortical excitability (upregulation), similar to long-term potentiation (LTP); this is due to incremental depolarization of membrane potentials. Combining tDCS with behavioral-based approaches has been found to enhance the learning process and increase the likelihood of retention. Specifically, tDCS has been shown to have a positive effect on cognitive functions in several studies in healthy humans, ${ }^{6}$ and in neuropsychiatric symptoms such as depression. ${ }^{7}$ Neuromodulation studies targeting cognition in non-Alzheimer dementias and mild cognitive impairment were almost nonexistent, ${ }^{8}$ although recent studies started to address other types, including Lewi Body Dementia (LBD) ${ }^{9}$ and FTD. ${ }^{10}$ The conceptual framework is that by improving plasticity, tDCS becomes a promising approach to reduce cognitive decline. As such, a recent study using 30 minutes of anodal $(2 \mathrm{~mA})$ tDCS stimulation applied to the parietal lobe (P3) showed some improvement in anomia, AD, and FTD patients, which demonstrates significant relevance for two-months post stimulation. ${ }^{11}$ Another investigation aimed to assess memory changes after five consecutive sessions of bilateral temporal anodal tDCS in patients with $\mathrm{AD}$; this shows significant improvement in visual recognition memory for at least four weeks after stimulation. ${ }^{12}$ A bilateral frontotemporal anodal stimulation $(2 \mathrm{~mA}, 20 \mathrm{~min}$, five consecutive days) showed improvement of behavioral scores in FTD patients. ${ }^{13}$ A second cross-over double-blind study with AD and FTD patients
Volume 9 Issue I - 2019

\author{
Inês Tello RM Rodrigues \\ Institute of Health Sciences, Universidade Católica Portuguesa, \\ Portugal
}

Correspondence: Inês Tello RM Rodrigues, Center of Interdisciplinary Investigation in Health, Institute of Health Sciences, Universidade Católica Portuguesa - Lisbon, Portugal, Email inestellorodrigues@gmail.com

Received: June 23, 2018 | Published: January 29, 2019

revealed improvements in picture naming. ${ }^{14} \mathrm{~A}$ specific study with a variant of FTD (primary progressive aphasia) used anodal tDCS applied to the left dorsolateral prefrontal cortex during individualized computerized anomia training, along with a positive effect in object naming. ${ }^{15}$ Another study with FTD patients aimed at a similar outcome (verbal fluency), but failed to convey any beneficial effect. ${ }^{16}$

\section{Conclusion}

The general outlines are promising, but the number of studies investigating the outcomes of tDCS on dementia is limited, especially with regard to clinically meaningful long-term effects. Additionally, most clinical trials are comprised of inadequate control arms and small samples, with few to no data reported in several dementia groups (e.g., VaD, LBD, and PDD). Several challenges exist in terms of stimulation parameters, a specific montage, and sample selection to establish clinical significance. Nevertheless, the continuous progress and optimization of stimulation protocols might soon provide a different and alternative therapeutic paradigm for symptom management in dementia patients.

\section{Acknowledgments}

None.

\section{Conflicts of interest}

The authors declare no conflicts of interest.

\section{References}

1. Wimo A, Guerchet M, Ali GC, et al. The worldwide costs of dementia 2015 and comparisons with 2010. Alzheimers Dement. 2017;3(1):1-7.

2. Sink KM, Holden KF, Yaffe K. Pharmacological treatment of neuropsychiatric symptoms of dementia: a review of the evidence. JAMA. 2006;7(3):201-202.

3. Stagg CJ, Nitsche MA. Physiological basis of transcranial direct current stimulation. Neuroscientist. 2011;17(1):37-53.

4. Nitsche MA, Liebetanz D, Lang N, et al. Safety criteria for transcranial direct current stimulation (tDCS) in humans. Clin Neurophysiol. 2003;114(11):2220-2222. 
5. Miranda PC, Mekonnen A, Salvador R, et al. The electric field in the cortex during transcranial current stimulation. Neuroimage. 2013;70:4858.

6. Kuo MF, Nitsche MA. Effects of transcranial electrical stimulation on cognition. Clin EEG Neurosci. 2012:3(3):192-199.

7. Loo CK, Alonzo A, Martin D, et al. Transcranial direct current stimulation for depression: 3-week, randomised, sham-controlled trial. Br J Psychiatry. 2012;200(1):52-59.

8. Elder GJ, Taylor JP. Transcranial magnetic stimulation and transcranial direct current stimulation: treatments for cognitive and neuropsychiatric symptoms in the neurodegenerative dementias? Alzheimers Res Ther 2014;6(5):74.

9. Elder GJ, Firbank MJ, Kumar H, et al. Effects of transcranial direct current stimulation upon attention and visuoperceptual function in Lewy body dementia: a preliminary study. Int Psychogeriatr. 2016;28(02):341-347.

10. Roncero C, Kniefel H, Service E, et al. Inferior parietal transcranial direct current stimulation with training improves cognition in anomic Alzheimer's disease and frontotemporal dementia. Alzheimers Dement. 2017;3(2):247-253.

11. Chertkow H, Roncero C, Kneifel H, et al. Transcranial direct current stimulation (tDCS) improves picture naming in Alzheimer Disease and Frontotemporal dementia.(P3. 089). Neurology. 2017;88(16 Supplement).
12. Boggio PS, Ferruci R, Mameli F, et al. Prolonged visual memory enhancement after direct current stimulation in Alzheimer's disease. Brain Stimul. 2012;5(3):223-230.

13. Ferrucci R, Ruggieroa F, Vergaria M, et al. ID 303-Transcranial direct current stimulation (tDCS) in patients with frontotemporal dementia. Clinical Neurophysiology. 2016;127(3):e100.

14. Roncero CT, Chertkow H, Vogt H, et al. Transcranial Direct Current Stimulation To The Left Parietal Lobe Combined With Training Improves Naming And Digit Span In People With Dementia. Alzheimer's \& Dementia: The Journal of the Alzheimer's Association. 2016;12(7):P258.

15. Cotelli M, Manenti R, Paternicò D, et al. Grey Matter Density Predicts the Improvement of Naming Abilities After tDCS Intervention in Agrammatic Variant of Primary Progressive Aphasia. Brain topogr. 2016;29(5):738-751.

16. Huey ED, Probasco JC, Moll J, et al. No effect of DC brain polarization on verbal fluency in patients with advanced frontotemporal dementia. Clin Neurophysiol. 2007;118(6):1417-1418. 